\title{
An in-depth look into PTSD-depression comorbidity: A longitudinal study of chronically-exposed Detroit residents
}

\author{
Danny Horesh $^{\mathrm{a}, *}$, Sarah R. Lowe ${ }^{\mathrm{b}}$, Sandro Galea ${ }^{\mathrm{c}}$, Allison E. Aiello ${ }^{\mathrm{d}}$, Monica Uddin ${ }^{\mathrm{e}}$, \\ Karestan C. Koenen ${ }^{f}$ \\ a Department of Psychology, Bar-Ilan University \& Depratment of Psychiatry, NYU School of Medicine \\ b Department of Psychology, Montclair State University \\ c Boston University School of Public Health \\ d Gillings School of Global Public Health, University of North Carolina at Chapel Hill \\ e Department of Psychology, University of Illinois at Urbana-Champaign \\ ${ }^{\mathrm{f}}$ Department of Epidemiology, Harvard T.H. Chan School of Public Health
}

\section{A R T I C L E I N F O}

\section{Keywords:}

PTSD

Depression

Comorbidity

Neighborhoods

Longitudinal study

\begin{abstract}
A B S T R A C T
Background: Although PTSD-major depressive disorder (MDD) co-morbidity is well-established, the vast majority of studies have examined comorbidity at the level of PTSD total severity, rather than at the level of specific PTSD symptom clusters. This study aimed to examine the long-term associations between MDD and PTSD symptom clusters (intrusion, avoidance, hyperarousal), and the moderating role of gender in these associations.

Methods: 942 residents of urban Detroit neighborhoods were interviewed at 3 waves, 1 year apart. At each wave, they were assessed for PTSD, depression, trauma exposure, and stressful life events.

Results: At all waves, hyperarousal was the PTSD cluster most strongly correlated with MDD. For the full sample, a reciprocal relationship was found between MDD and all three PTSD clusters across time. Interestingly, the relative strength of associations between MDD and specific PTSD clusters changed over time. Women showed the same bidirectional MDD-PTSD pattern as in the entire sample, while men sometimes showed non-significant associations between early MDD and subsequent PTSD clusters.

Limitations: First, our analyses are based on DSM-IV criteria, as this was the existing edition at the time of this study. Second, although this is a longitudinal study, inferences regarding temporal precedence of one disorder over another must be made with caution.

Conclusions: Early identification of either PTSD or MDD following trauma may be crucial in order to prevent the development of the other disorder over time. The PTSD cluster of hyper-arousal may require special therapeutic attention. Also, professionals are encouraged to develop more gender-specific interventions posttrauma.
\end{abstract}

\section{Introduction}

Numerous studies have shown that exposure to trauma often leads to significant psychiatric distress. The most common psychopathological outcome of trauma is post-traumatic stress disorder (PTSD). However, other psychiatric disorders may follow trauma exposure, including substance abuse, somatic disorders, anxiety disorders, and major depression (MDD), the latter found to be the most common post-trauma disorder other than PTSD (e.g. O'Donnell et al., 2004). Studies have shown high rates of PTSD-MDD comorbidity, with lifetime rates ranging from $43-56 \%$, and current rates from $16-37 \%$
(Breslau et al., 1997; Kulka et al., 1990; Norris et al., 2004).

Although PTSD-MDD comorbidity is well-established, not much is known about its nature and underlying mechanisms. Several competing models of comorbidity have been suggested. One model posits that PTSD is the "primary", antecedent disorder, which may cause subsequent MDD (Schindel-Allon et al., 2010). A second model, sometimes referred to as "the depressogenic model" (Schindel-Allon et al., 2010), suggests the opposite direction of causality, with initial MDD yielding subsequent PTSD. A third model postulates that both disorders may be independent manifestations of a larger post-traumatic "meta-factor" (e.g., negative affectivity, stress-related biological processes), which

\footnotetext{
* Corresponding author.

E-mail addresses: danny.horesh@biu.ac.il (D. Horesh), aaiello@email.unc.edu (A.E. Aiello), muddin@illinois.edu (M. Uddin), kkoenen@hsph.harvard.edu (K.C. Koenen).
} 
may yield both disorders (Breslau et al., 2000). Longitudinal studies focusing on various post-trauma samples have shown evidence supporting each of these models, with inconclusive results (e.g., Dekel et al., 2014; Horesh et al., 2015). However, the vast majority of these studies have referred to PTSD as a whole, without differentiating between its individual constituent symptom clusters and their respective associations with MDD over time (e.g., Itzhaky et al., 2014). Thus, while research on PTSD alone has been characterized by an increased focus on micro-level analyses, looking at specific clusters, symptoms and symptom profiles (e.g., Seligowski et al., 2016), the same cannot be said about comorbidity studies. The scarcity of this type of comorbidity studies is particularly apparent in light of the emerging RDoC approach (Insel et al., 2010), which encourages a deeper, trans-diagnostic examination of underlying dimensions of psychopathology.

Several cross-sectional studies have shown differential associations between PTSD symptom clusters and depression symptoms, yet findings have been mixed. For example, in a study by Rubacka et al. (2008), PTSD hyperarousal, but not avoidance or re-experiencing, was associated with chronic MDD. However, other cross-sectional studies have found avoidance/numbing (Gros et al., 2010) and re-experiencing (Post et al., 2011) to be the clusters most strongly associated with MDD symptoms. Thus, it still remains unclear which PTSD cluster is most strongly related to MDD. Not only do these studies present a mixed, unclear picture, they are also limited by their cross-sectional nature. Longitudinal studies on PTSD-MDD comorbidity, particularly at the level of specific symptom clusters, are therefore both rare and muchneeded.

In a previous paper published by our group (Horesh et al., 2015), we have longitudinally examined PTSD-MDD comorbidity among residents of urban Detroit, Michigan. For decades Detroit has been struggling with high crime rates and severe financial difficulties (Fisher, 2015). In our previous paper, we have found high rates of lifetime trauma exposure, PTSD and MDD, in line with previous studies of urban American neighborhoods (Galea et al., 2007; Horowitz et al., 1995).

Most studies to date have also failed to examine factors that may moderate PTSD-MDD associations over time. One variable that seems to hold promise in this regard is gender. While female gender was often found to be a risk factor for each of these disorders alone (Hourani et al., 2015), much less in known about its role in their comorbidity, particularly over time. In our previous paper, mentioned above, we reported that Detroit women suffered from higher levels of PTSD and MDD alone, as well as higher comorbidity rates across time. We also showed gender differences in comorbidity patterns over time between men and women.

In the present study, we aimed to expand our work from that previous study, by taking a closer, more in-depth look at PTSD-MDD comorbidity. We did so by examining each PTSD symptom cluster separately, and its temporal associations with MDD. This unique longitudinal examination of comorbidity at the level of symptom clusters aimed to shed light on the underlying mechanisms of comorbidity. More specifically, we addressed two major research questions:

1. What is the pattern (i.e, unidirectional/bidirectional) of long-term associations between MDD and the PTSD symptom clusters of reexperiencing, avoidance and hyperarousal among residents of innercity Detroit neighborhoods?

2. Is the temporal pattern of associations between MDD and PTSD clusters different for men and women?

We expected to find a complex pattern of findings, wherein the relative predictive dominance of PTSD clusters vis-à-vis MDD would change over time, with different temporal associations for male and female participants.

\section{Methods}

\subsection{Participants and procedure}

Data were collected as part of the Detroit Neighborhood Health Study (DNHS), a longitudinal study of predominantly non-Hispanic Black adults living in Detroit. Details on the baseline sampling methodology of DNHS can be found elsewhere (e.g., Uddin et al., 2010). Wave 1 (W1) was conducted between 2008 and 2009, and 1547 participants completed the survey. Approximately a year after W1, 1054 participants from W1 completed the Wave 2 (W2) survey. Wave 3 (W3) occurred approximately a year after W2, and 965 participants from W1 participated. A total of 847 participants completed all three waves, yielding an overall retention rate of $54.8 \%$. At each wave, participants completed a structured telephone survey using standardized instruments. Informed consent was obtained at the beginning of each survey, and participants were offered $\$ 25$ for their participation.

The current study included 942 participants who reported at least one lifetime traumatic event at W1, and completed the PTSD and MDD questionnaires at W1, and either or both W2 and W3 ( $N=942$; men: $n=387$ [41.1\%]; women: $n=555$ [58.9\%]). The majority of the participants (85.3\%) identified as non-Hispanic Black, $10.5 \%$ as nonHispanic White, and $1.2 \%$ as Hispanic. On average, participants were 52.63 years old ( $S D=16.02$; range: $18-92)$ at $\mathrm{W} 1$. At $\mathrm{W} 1,26.0 \%$ were married, whereas $25.7 \%$ were separated or divorced, $13.4 \%$ widowed, and $34.9 \%$ single and never married. Participants also reported on their income (from $1=<\$ 10,000$ to $7=>\$ 75,000 ; M=3.84, S D=2.00$ ), highest level of education (from $1=$ less than high school to $5=$ graduate degree; $M=2.78, S D=1.08$ ) and employment status (58.7\% unemployed) at W1. Bonferroni-corrected independent samples $t$-tests and chi-square tests showed that retained participants had significantly more lifetime traumatic events and stressors, significantly higher W1 and W2 depression, and were significantly more likely to be divorced or separated and less likely to be single and never married than dropped participants. In addition, for the 942 participants in the analysis, those with complete data $(n=269)$ reported significantly higher W2 reexperiencing, avoidance, and hyperarousal symptoms, and higher W2 and W3 depression than those missing data on any variable $(n=673)$.

\subsection{Measures}

\subsubsection{Lifetime traumatic events and stressors}

At W1, participants completed a 20 -item trauma inventory, where they indicated whether they had experienced 19 traumatic events (e.g., rape, serious car or motor vehicle crash) in their lifetime (Breslau et al., 1998), as well as an additional item allowing participants to report another traumatic event not on the inventory. A count of affirmative responses was created as an index of lifetime traumatic events exposure. Participants also indicated whether they experienced 10 stressors (e.g., serious financial problem, divorce) in their lifetime, and a count of affirmative responses was included as an index of lifetime stressors. As reported in our previous paper, lifetime trauma exposure rates were very high. For example: $37.7 \%$ (men - $47 \%$, women - $31.2 \%$ ) have been mugged, held up or threatened with a weapon; $32.1 \%$ (men - 42.9\%, women $-24.5 \%$ ) have witnessed someone being killed or seriously injured; $11.9 \%$ (men $-1.8 \%$, women $-18.9 \%$ ) were raped; $29 \%$ (men $-30.5 \%$, women $-27.9 \%$ ) were in a serious car or motor vehicle crash. For a comprehensive list of all lifetime traumas and stressors, please see Horesh et al. (2015).

\subsubsection{Posttraumatic stress}

A modified interview version of the PTSD Checklist-Civilian Version (Weathers et al., 1996), validated by the Clinician Administered PTSD Scale (CAPS; Blake et al., 1995), assessed PTSD symptoms at each wave. The PCL-C includes 17 items, representing criteria B (reexperiencing; five items), C (avoidance; seven items), and D (hyperar- 
ousal; five items) from DSM-IV-TR (APA, 2000). At each assessment, participants were asked to respond based on the event reported as the "worst" at W1. Participants indicated the degree to which they had been bothered by each symptom as a result of the event since the last interview, from $1=$ Not at all to $5=$ Extremely. Responses were summed to yield a severity score for each symptom cluster, ranging from 5 to 25 for re-experiencing and hyperarousal, and 7-35 for avoidance (for W1-W3: re-experiencing: $\alpha=.82-.86$; avoidance: $\alpha=.83-.87$; hyperarousal: $\alpha=.86-.87$ ).

\subsubsection{Depression}

A modified interview version of the nine-item Patient Health Questionnaire-9 (PHQ-9; Kroenke and Spitzer, 2002) assessed depression symptoms at each wave. Participants rated the frequency (from 0 - not at all, to 3 - nearly every day) in which they were bothered by each depression symptom since the last interview, over a period of two weeks. The total PHQ-9 score was created by summing ratings for all symptoms, with a possible range of $0-27$. Previous studies have found the PHQ-9 to have excellent internal consistency, test-retest reliability, and construct validity (Kroenke et al., 2001) (for W1-W3: $\alpha=.85-.86$ ).

\subsection{Data analysis}

Among the variables included in the study, the missing rate ranged from $0-49.9 \%$, and the overall missing rate was $11.7 \%$. We conducted multiple imputation in Amelia II for R to handle missing data, and five complete datasets were used for statistical analyses. Results represent an average of the five separate analyses with Rubin's (1987) correction of standard error.

The first stage of data analysis was conducted in SPSS 19.0. Means and SDs for the PCL-C subscales and PHQ-9 were computed for the full sample and male and female subsamples, and independent-samples $t$ tests were conducted to assess gender differences. Second, correlations between PHQ-9 and the PCL-C subscales were computed for the full sample and stratified by gender. Fisher $r$-to- $z$ transformations were used to determine statistical significance of observed differences in correlations. The same method assessed for gender differences in each of the correlations at each wave. Third, repeated measures analyses of variance were conducted to assess linear and quadratic change in each PCL-C subscale and PHQ-9 over the study among participants in the full sample and male and female subsamples.

The second stage of data analysis was conducted in Mplus 6.0. Cross-lagged models were run to determine the association of each PCL-C subscale at one wave with the PHQ-9 score at the subsequent wave, and vice versa, over time. Cutoffs for acceptable model fit were set at <.08 for RMSEA and > .90 for CFA (Hu and Bentler, 1998). The first set of cross-lagged models included all study participants. Demographic characteristics (e.g. age, gender, ethnicity) and counts of lifetime traumatic events and stressors were included as predictors of W1 PCL-C and PHQ-9, and any paths with $\mathrm{p}>.10$ were trimmed. Subsequently, multigroup cross-lagged models by gender, which included the same set of trimmed covariates except for female gender, were conducted to explore whether cross-lagged paths differed between male and female participants. Separate models with equality constraints for each path (e.g., the path from W1 PCL-C re-experiencing to W2 PHQ-9 constrained to be equal for men and women) were computed. Chi-square difference tests were conducted to determine whether the constraints led to significantly worse fit.

\section{Results}

\subsection{Levels of MDD and PTSD clusters}

Table 1 presents means and standard deviations for the PHQ-9 and PCL-C subscales for full sample and male and female subsamples at each wave, and the results of independent-samples $t$-tests assessing
Table 1

Means and standard deviations for the PHQ-9 and PCL-C subscales and results of independent-samples $t$-tests of gender differences.

\begin{tabular}{|c|c|c|c|c|}
\hline & $\begin{array}{l}\text { Full sample } \\
(\mathrm{N}=942)\end{array}$ & $\begin{array}{l}\text { Men } \\
(n=387)\end{array}$ & $\begin{array}{l}\text { Women } \\
(n=555)\end{array}$ & $\begin{array}{l}\text { Gender } \\
\text { difference }(t)\end{array}$ \\
\hline \multicolumn{5}{|l|}{$P H Q-9$} \\
\hline Wave 1 & $5.69(6.30)$ & $\begin{array}{l}4.77 \\
(6.11)\end{array}$ & $6.31(6.35)$ & $-3.41^{* * * *}$ \\
\hline Wave 2 & $4.32(5.55)$ & $\begin{array}{l}3.69 \\
(5.32)\end{array}$ & $4.77(5.64)$ & $-3.35^{* * * * *}$ \\
\hline Wave 3 & $4.10(5.23)$ & $\begin{array}{l}3.19 \\
(5.02)\end{array}$ & $4.63(5.80)$ & $-3.51^{* * * a}$ \\
\hline \multicolumn{5}{|c|}{$P C L-C$ re-experiencing } \\
\hline Wave 1 & $10.93(5.08)$ & $\begin{array}{l}10.04 \\
(4.64)\end{array}$ & $\begin{array}{l}11.55 \\
(5.28)\end{array}$ & $-4.63^{* * * a}$ \\
\hline Wave 2 & $8.12(3.73)$ & $\begin{array}{l}7.89 \\
(3.57)\end{array}$ & $8.29(3.84)$ & -1.64 \\
\hline Wave 3 & $8.28(4.08)$ & $\begin{array}{l}7.66 \\
(3.89)\end{array}$ & $8.72(4.15)$ & $-3.94^{* * * * *}$ \\
\hline \multicolumn{5}{|l|}{ PCL-avoidance } \\
\hline Wave 1 & $12.81(6.54)$ & $\begin{array}{l}12.19 \\
(6.14)\end{array}$ & $\begin{array}{l}13.25 \\
(6.77)\end{array}$ & $-2.50^{* a}$ \\
\hline Wave 2 & $10.74(5.15)$ & $\begin{array}{l}10.64 \\
(5.14)\end{array}$ & $\begin{array}{l}10.81 \\
(5.16)\end{array}$ & -.50 \\
\hline Wave 3 & $10.23(5.08)$ & $\begin{array}{l}9.75 \\
(4.88)\end{array}$ & $1.55(5.19)$ & $-2.38^{\mathrm{a}}$ \\
\hline \multicolumn{5}{|l|}{ PCL-C hyperarousal } \\
\hline Wave 1 & $9.85(5.00)$ & $\begin{array}{l}9.04 \\
(4.45)\end{array}$ & $\begin{array}{l}10.42 \\
(5.27)\end{array}$ & $-4.32^{* * * *_{a}}$ \\
\hline Wave 2 & $8.66(4.27)$ & $\begin{array}{l}8.34 \\
(4.06)\end{array}$ & $8.88(4.41)$ & 1.90 \\
\hline Wave 3 & $8.65(4.44)$ & $\begin{array}{l}8.27 \\
(4.15)\end{array}$ & $8.92(4.61)$ & $-2.25^{*, \mathrm{a}}$ \\
\hline
\end{tabular}

Note.

${ }^{*} p<.05$,

**** $p<.001$

${ }^{a}$ Equal variances not assumed due to significant Levene's test. Adjusted degrees of freedom were 889 for Wave 3 PHQ-9, 891 for Wave 1 PCL-C re-experiencing, 878 for Wave 1 PCL-C avoidance, 906 for Wave 1 PCL-C hyperarousal, and 881 for Wave 3 PCLC hyperarousal.

gender differences. As shown, female participants had significantly higher PHQ-9 scores than male participants at each wave. Women also had higher scores on each PCL-C subscale at W1 and W3, whereas there were no significant gender differences on the PCL-C subscales at W2.

\subsection{Correlations between the PHQ-9 and PCL-C subscales}

Table 2 lists correlations between the PHQ-9 and each PCL-C subscale at each wave for the full sample and for men and women separately.

Each correlation reached statistical significance; however, there was significant variation among the correlations between the PHQ-9 and the three PCL-C subscales. At W1, the PHQ-9 was significantly more strongly correlated with hyperarousal than with re-experiencing for the full sample, and the same was true for both the male and female subsamples. At W2, the PHQ-9 was significantly more strongly correlated with hyperarousal than with re-experiencing for the full sample and the female subsample, and also more strongly correlated with avoidance than with re-experiencing for the female subsample. In contrast, there were no significant differences among the correlations at W2 for men. At W3, the PHQ-9 was significantly more strongly correlated with hyperarousal and avoidance than with re-experiencing for the full sample, and the same was true for both the male and female subsamples. There were no significant gender differences in the individual correlation coefficients. 
Table 2

Correlations between the PHQ-9 and PCL-C subscales at each wave.

\begin{tabular}{|c|c|c|c|c|c|c|c|}
\hline & & \multicolumn{2}{|c|}{ Full sample $(N=942)$} & \multicolumn{2}{|c|}{ Men $(n=387)$} & \multicolumn{2}{|c|}{ Women $(n=555)$} \\
\hline & & $r$ & Comparisons & $r$ & Comparisons & $r$ & Comparisons \\
\hline \multirow[t]{4}{*}{ Wave 1} & & & $\mathrm{H}>\mathrm{R}^{* * * *}$ & & $\mathrm{H}>\mathrm{R}^{* * *}$ & & $\mathrm{H}>\mathrm{R}^{*}$ \\
\hline & Re-experiencing (R) & $.48^{* * * *}$ & & $.44^{* * * *}$ & & $.48^{* * * *}$ & \\
\hline & Avoidance (A) & $.53^{* * * * *}$ & & $.51^{* * * * *}$ & & $.53^{* * * *}$ & \\
\hline & Hyperarousal (H) & $.59^{* * * *}$ & & $.60^{* * * *}$ & & $.58^{* * * * *}$ & \\
\hline \multirow[t]{4}{*}{ Wave 2} & & & $\mathrm{H}>\mathrm{R}^{* *}$ & & - & & $\mathrm{H}>\mathrm{R}^{* * *} ; \mathrm{A}>\mathrm{R}^{*}$ \\
\hline & Re-experiencing (R) & $.51^{* * * *}$ & & $.52^{* * * * *}$ & & $.48^{* * * *}$ & \\
\hline & Avoidance (A) & $.58^{* * * *}$ & & $.57^{* * * *}$ & & $.58^{* * * *}$ & \\
\hline & Hyperarousal (H) & $.61^{* * * * *}$ & & $.61^{* * * *}$ & & $.60^{* * * * *}$ & \\
\hline \multirow[t]{4}{*}{ Wave 3} & & & $\mathrm{H}>\mathrm{R}^{* * * *} ; \mathrm{A}>\mathrm{R}^{* * * *}$ & & $\mathrm{H}>\mathrm{R}^{* * *}, \mathrm{~A}>\mathrm{R}^{* *}$ & & $\mathrm{H}>\mathrm{R}^{* * * *} ; \mathrm{A}>\mathrm{R}^{* * * *}$ \\
\hline & Re-experiencing (R) & $.49^{* * * *}$ & & $.49^{* * * * *}$ & & $.48^{* * * *}$ & \\
\hline & Avoidance (A) & $.65^{* * * *}$ & & $.63^{* * * *}$ & & $.65^{* * * * *}$ & \\
\hline & Hyperarousal (H) & $.67^{* * * *}$ & & $.62^{* * * *}$ & & $.69^{* * * * *}$ & \\
\hline
\end{tabular}

Note. Comparisons of the correlations between the PHQ- 9 and the three PCL-C subscales were conducted using Fisher $r$-to- $z$ transformations.

${ }^{*} p<.05$

$p<.01$,

$p<.001$.

\subsection{How are PCL-C subscales and PHQ-9 changing over time?}

Table 3 shows the results of repeated-measures ANOVA for the PHQ-9 and the three PCL-C subscales for the full sample and male and female subsamples. Average trajectories for each measure are depicted in Fig. 1.

For the full sample, each measure was characterized by significant linear and quadratic growth. Inspection of means showed that the PHQ-9 and the PCL-C subscales each decreased from W1 to W2, and were relatively stable from W2 to W3. The same patterns of statistical significance were detected for the male and female subsamples, with one exception: the quadratic term for avoidance in the male subsample was non-significant. It was also observed that, across all four measures, the quadratic trends were much stronger for women than men. Inspection of means showed men had more consistent declines on average than women on each measure.

\subsection{What is the nature of the associations between PCL-C subscales and $P H Q-9$ ?}

The cross-lagged models for the full sample each had acceptable fit with the data (see Fig. 2).

Table 3

Results of repeated measures analyses of variance (ANOVAs) for the PHQ-9 and PCL-C subscales.

\begin{tabular}{|c|c|c|c|}
\hline & $\begin{array}{l}\text { Full sample } \\
(N=942)\end{array}$ & $\begin{array}{l}\text { Men } \\
(n=387)\end{array}$ & $\begin{array}{l}\text { Women } \\
(n=555)\end{array}$ \\
\hline \multicolumn{4}{|l|}{$P H Q-9$} \\
\hline Linear & $71.06^{* * * *}$ & $22.17^{* * *}$ & $49.70^{* * * *}$ \\
\hline Quadratic & $16.13^{* * * *}$ & $4.51^{*}$ & $12.26^{* * * *}$ \\
\hline \multicolumn{4}{|c|}{$P C L-C$ re-experiencing } \\
\hline Linear & $150.23^{* * * *}$ & $63.45^{* * * *}$ & $113.23^{* * * *}$ \\
\hline Quadratic & $127.60^{* * * *}$ & $31.81^{* * * *}$ & $113.53^{* * * *}$ \\
\hline \multicolumn{4}{|l|}{$P C L-C$ avoidance } \\
\hline Linear & $120.38^{* * * * *}$ & $46.67^{* * * *}$ & $83.70^{* * * *}$ \\
\hline Quadratic & $33.63^{* * * *}$ & 3.33 & $34.86^{* * * *}$ \\
\hline \multicolumn{4}{|l|}{ PCL-C hyperarousal } \\
\hline Linear & $5.56^{* * * *}$ & $11.97^{* * *}$ & $41.81^{* * * *}$ \\
\hline Quadratic & $26.93^{* * * *}$ & $4.38^{*}$ & $24.71^{* * * *}$ \\
\hline
\end{tabular}

Note. Values are the $F$ statistic. Degrees of freedom were 1, 941 for the full sample; 1, 386 for the male subsample; and 1, 941 for the female subsample.

${ }^{*} p<.05$.

${ }^{* * *} p<.01$.

$p<.001$.
All cross-lagged paths were statistically significant, thus indicating a bidirectional association between each PTSD cluster and MDD across time (i.e., a given PTSD cluster at one wave predicted MDD at the next wave, and vice versa). Individual path coefficients were inspected to assess variation in the cross-lagged paths over time. For each PCL-C subscale, the path from PHQ-9 to PCL-C was smaller between W1 and W2 than between W2 and W3. Constraining the PHQ-9 to reexperiencing paths as equal over time led to a significant decline in fit, $\chi^{2} \Delta(1)=12.19, p<.001$. In contrast, there were no consistent patterns among the PCL-C subscales in the paths from PCL-C to PHQ-9, and in no case did equality constraints over time lead to significant worse fit.

Path coefficients across the three PCL-C subscale models were also compared, although equality constraints could not be added to test whether any observed differences reached statistical significance, as the models were not nested. The path from W1 PHQ-9 to W2 PCL-C was strongest for hyperarousal (.18) and weakest for re-experiencing (.10). In contrast, the path from W2 PHQ-9 to W3 PCL-C was strongest for re-experiencing (.26) and weakest for avoidance (.17). Whereas no notable differences among the PCL-C subscales were observed for the path from W1 PCL-C to W2 PHQ-9, the path from W2 PCL-C to W3 PHQ-9 was strongest for avoidance (.20) and weakest for re-experiencing (.14).

The multigroup cross-lagged models for male and female participants are shown in Fig. 3.

Each model had acceptable fit with the data. All cross-lagged paths reached statistical significance, with three exceptions: for male participants, both paths from PHQ-9 to avoidance were marginally significant, as was the path from W2 PHQ-9 to W3 hyperarousal. Thus, for females, a bidirectional pattern of associations appeared across time, but this was only partially true for men. Also, the paths from PHQ-9 to PCL-C subscales tended to be weaker for men than for women. Specifically, the W1 PHQ-9 to W2 PCL-C path was weaker for men than women in the avoidance and hyperarousal models, and the W2 PHQ-9 to W3 PCL-C path was weaker for men for all three PCL-C subscale models. Different patterns were also observed in the paths from PCL-C subscales to PHQ-9. The W1 PCL-C to W2 PHQ-9 path appeared stronger for men than women in the re-experiencing model (.20 vs. 16), but weaker for men than women in the avoidance model (.16 vs. 21). In contrast, the W2 PCL-C to W3 PHQ-9 path was observed as stronger for men than women in both the re-experiencing and avoidance models (.19 vs. 11 , and .24 vs. 18 , respectively). Despite these observed gender differences, in no case did constraining individual cross-lagged paths to be equal across men and women lead to a 
PHQ-9

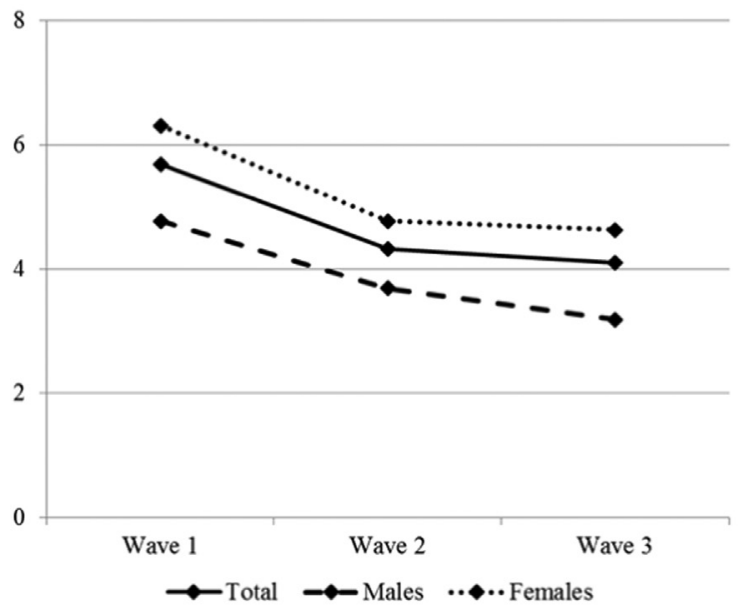

PCL-C Avoidance

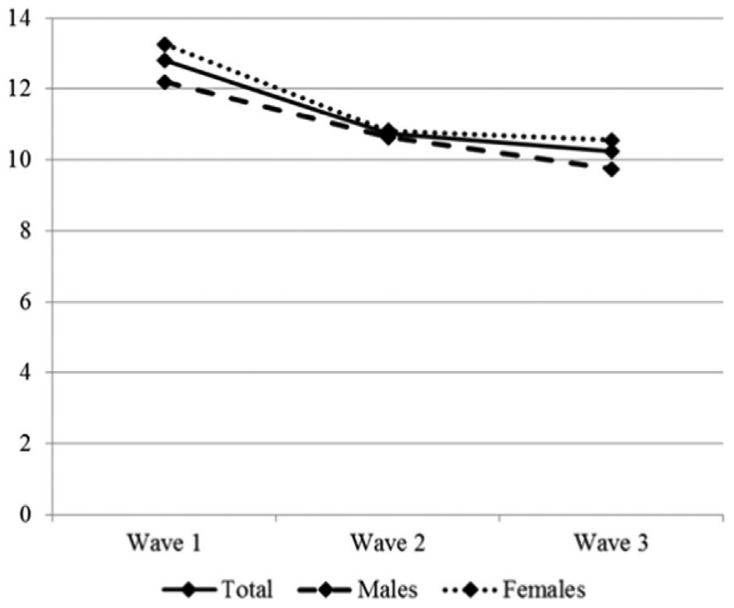

PCL-C Re-experiencing

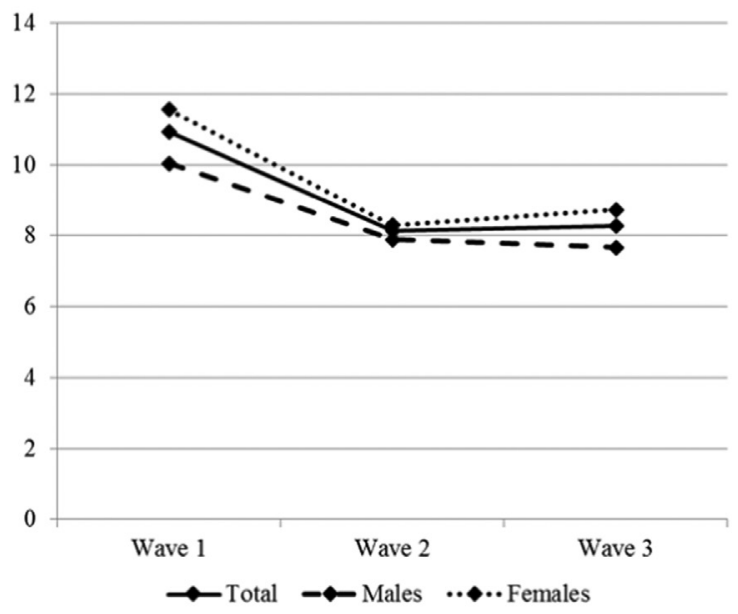

PCL-C Hyperarousal

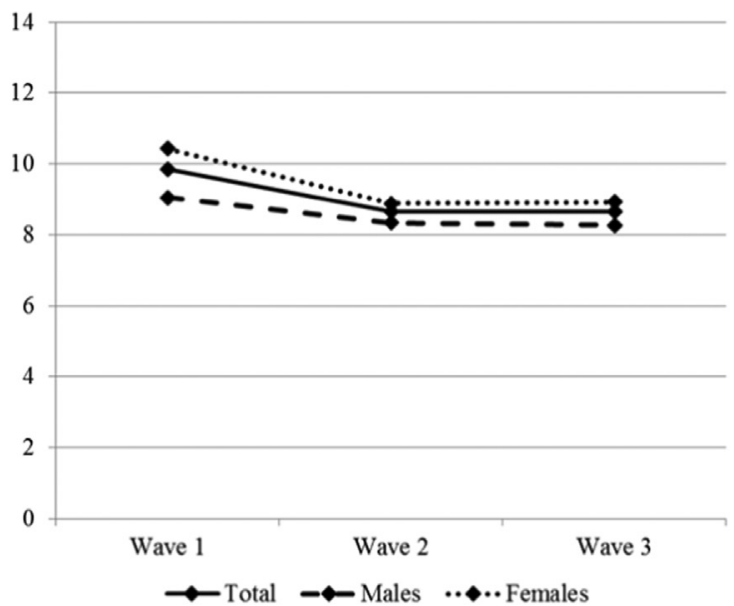

Fig. 1. Average trends in PHQ-9 and PCL-C subscales for the full sample ( $N=942)$, Men $(n=357)$, and Women ( $n=555)$.

significant decline in model fit.

\section{Discussion}

This study set out to examine gender differences in the long-term associations between MDD and PTSD symptom clusters among Detroit residents. At all three waves, hyperarousal was the PTSD cluster most strongly correlated with MDD. For the entire sample, bidirectional associations were found between MDD and PTSD avoidance, reexperiencing and hyperarousal, indicating a reciprocal relationship between the symptoms of both disorders across time. Interestingly, however, the relative strength of associations between MDD and PTSD clusters changed over time, with different clusters showing predictive dominance vis-à-vis MDD, and vice versa, at different waves. Thus, a complex temporal pattern of comorbidity appeared. Gender differences were also found, with women reporting higher levels of MDD at each wave, as well as higher levels of all three PTSD clusters at W1 and W3. Longitudinally, women showed the same bidirectional MDD-PTSD pattern as in the entire sample, while men often showed nonsignificant associations between early MDD and subsequent PTSD clusters.

In our previous paper based on this data (Horesh et al., 2015), we reported high rates of lifetime trauma exposure among those residing in urban Detroit neighborhoods. These findings reflected the difficult, chronically-stressful reality of many large American cities today. As previously suggested by others (Myers et al., 2015), these high exposure rates may be seen as representing a "compounded community trauma" (Horowitz, 1995), in which the traumatic whole is larger than the sum of its parts. In our previous paper, we have also reported high rates of both PTSD and MDD (21.5\% and 23.6\% of PTSD and MDD, respectively, at wave 1 , with rates subsequently declining). In our current analysis, we have found that all three PTSD symptom clusters, as well as MDD, declined between W1 and W2, and then stabilized between W2 and W3. This finding may be attributed to several factors. First, it provides further evidence for the strong associations between MDD and PTSD, as both disorders share a similar overall temporal trajectory. Second, this pattern may indicate spontaneous recovery/ remission with time, in line with previous studies of both PTSD (Koren et al., 2001) and MDD (Bockting et al., 2015). Finally, the finding showing reduced PTSD symptoms may be the result of a reporting bias. As noted, at W1 participants were asked to rate PTSD in reference to their "worst" reported trauma. They were then asked to continue to refer to that same trauma at W2 and W3, but may have been less emotionally connected to it than when they first reported it.

In our previous paper, we reported a bidirectional long-term association between MDD and PTSD. In the present study, we set out to elaborate this finding, and to understand whether specific elements within PTSD may be more or less responsible for the reciprocal long-term "fueling" between both disorders. Our findings indicate that this bidirectional association is maintained for all three PTSD symptom clusters. This finding strengthens our previouslyreported findings, and serves as further confirmation for the reciprocity between PTSD and MDD. Thus, in our sample, neither disorder was "secondary" to the other, but rather both seemed to act as possible 

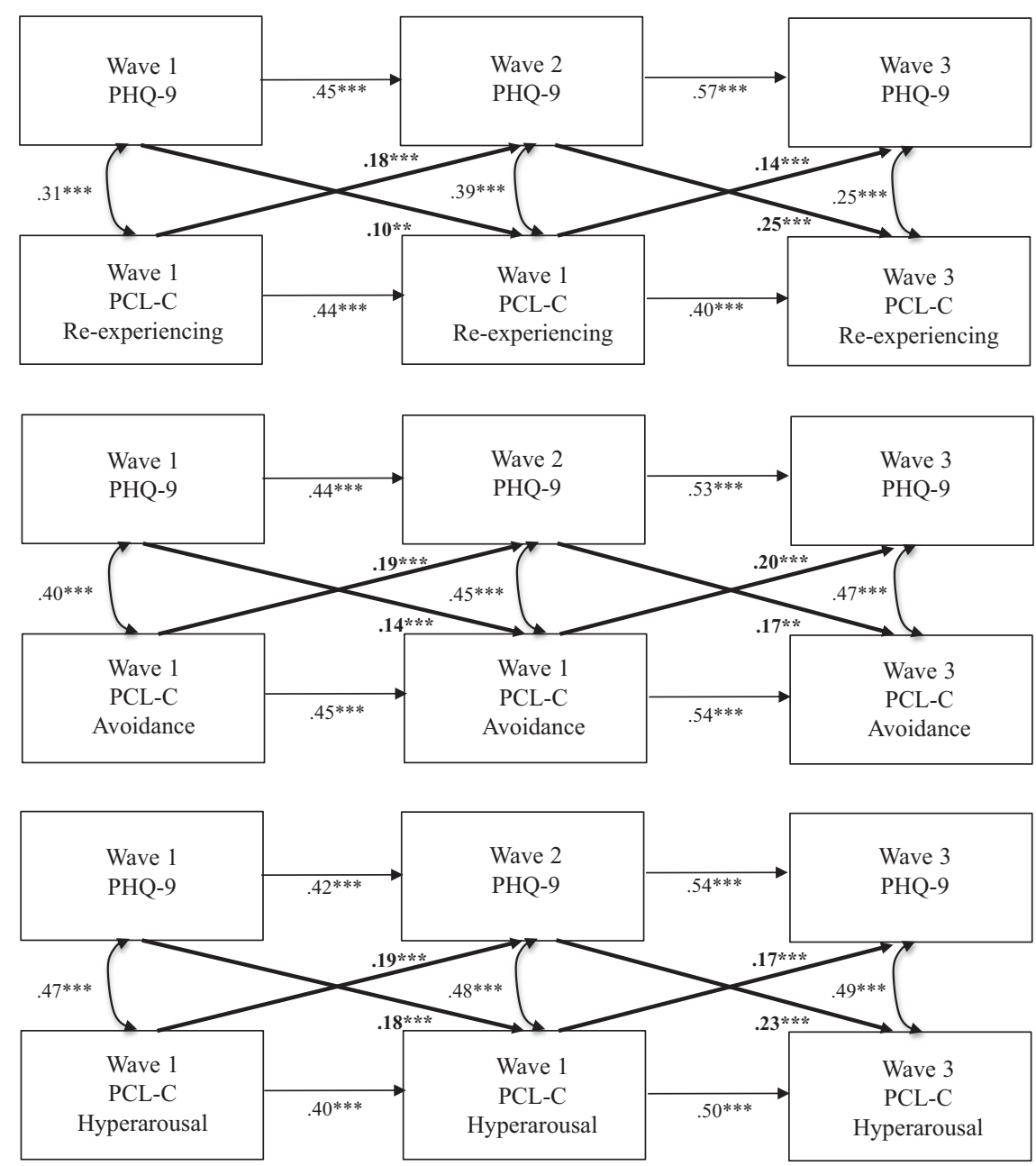

Fig. 2. Results of cross-lagged models between the PHQ- 9 and PCL-C subscales for the full sample $(N=942)$.

gateways into post-traumatic distress (Ginzburg et al., 2010).

Our finding that all three PTSD symptom clusters show a bidirectional association with MDD may be attributed to two main factors. First, PTSD was often found to be a unitary and clinically-coherent disorder, in which symptom clusters tend to develop in a parallel fashion over time (i.e., they increase and decrease as one block). This was found in both epidemiological (Solomon et al., 2009) and clinical (Horesh et al., 2016) studies. Put differently, PTSD clusters, despite representing varied clinical phenomena, seem to "behave" in a similar manner over time. At least according to our findings, this includes a rather consistent pattern of comorbidity with MDD. There are several explanations for MDD-PTSD comorbidity in general, among them: negative affect and negative cognitive schemas as shared elements; MDD resulting from the burden of living with PTSD; MDD preventing help-seeking and therefore yielding PTSD (O'Donnell et al., 2004). However, there are also more specific explanations for the association between MDD and each individual PTSD cluster. For example, MDD and PTSD avoidance share core features such as constricted affect and defensive attempts to ward-off unpleasant thoughts and emotions (Kashdan et al., 2009), while MDD and PTSD re-experiencing may be associated through negative cognitive schemas (Elwood et al., 2009). With such dominant shared factors, both MDD and PTSD may play a role as the primary, early disorder post-trauma, which later fuels the other disorder, as seen in our cross-lagged models.

Nonetheless, despite the fact that all three clusters showed a reciprocal association with MDD, an in-depth look reveals a more complex picture. First, at each wave, hyperarousal showed the strongest associations with MDD. This finding may have several explana- tions. First, our study focused on a community sample of Detroit residents, many of whom are likely chronically exposed to severe stressors. In environments where violence, poverty and crime prevail at levels higher than the general population, one may be forced to maintain alertness, in fear of further affliction. Thus, the symptoms included in the hyperarousal cluster (e.g., hypervigilance, startle responses) may be particularly common and distressing in such circumstances. A second explanation has to do with the underlying symptom structure of both PTSD and MDD. In recent years, several studies have examined the associations between broad symptomatic factors comprising both disorders. For example, in a study by Price and van Stolk-Cooke (2015), the affective factor of MDD was most strongly related to the emotional numbing factor of PTSD, whereas the somatic factor of MDD was most strongly related to the hyperarousal factor of PTSD. This association between PTSD hyper-arousal and somatic MDD symptoms, such as sleep changes and appetite disturbances, was also found in other studies (Biehn et al., 2013). While we did not specifically examine this question, it is possible that in our sample somatic MDD symptoms were more dominant. This issue calls for further study. A third explanation, which perhaps stems from the former one, is that hyperarousal represents a non-specific group of symptoms that are shared by several mood, anxiety and stress-related disorders (GreavesLord et al., 2007). As such, it may cut across multiple psychiatric diagnoses. In an attempt to explain shared symptoms of hyperarousal between PTSD, MDD and irritable bowel syndrome following early trauma, Kendall-Tackett (2000) addresses the biological processes associated with this symptom cluster. More specifically, she notes the effects of traumatic stress on the central nervous system, such as 

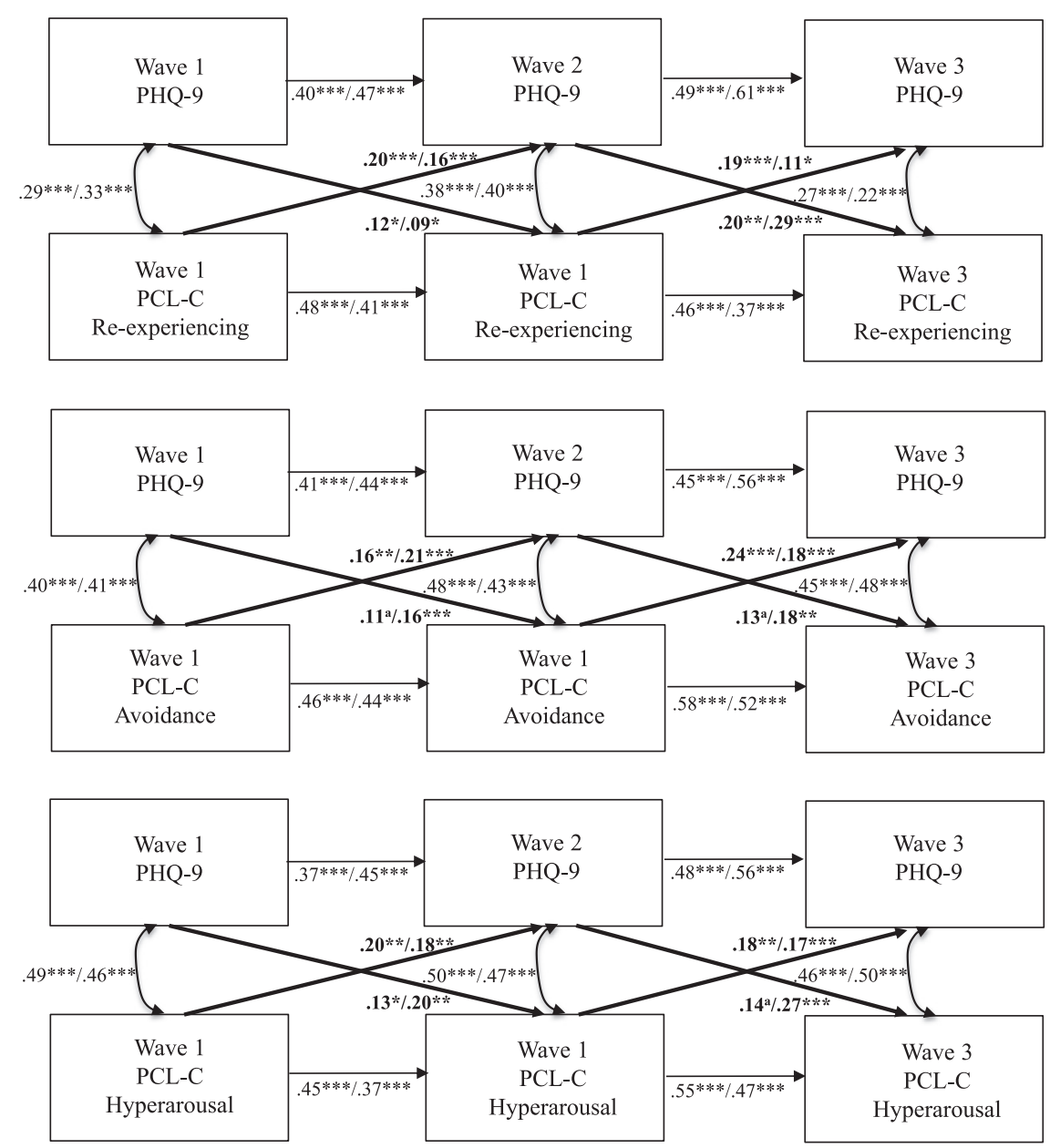

Fig. 3. Results of multigroup cross-lagged models by gender between the PHQ- 9 and PCL-C subscales for the full sample ( $N=942$; males: $n=357$; females: $n=555$ ).

alterations in frontal-limbic connections and alterations in feedback to the central nervous system from the brainstem aminergic nuclei, including the locus coeruleus and raphe nuclei. Also addressed is the association between traumatic exposure and a hypersensitive amygdala. Such processes have a significant effect on the modulation of arousal levels, which are shared by several disorders post-trauma (Lovallo, 1997). To sum, rather than being a disorder-specific symptom, hyperarousal may serve as a common psychopathological denominator that often underlies the association between otherwise distinct disorders, including PTSD and MDD.

The more complex aspects of comorbidity in our sample are also revealed longitudinally. Across time, early PTSD symptom clusters "switch turns" in best predicting MDD, and vice versa. Thus, the driving forces of PTSD-MDD comorbidity are labile and change over time. This perhaps should come as no surprise, as PTSD symptoms are known to wax and wane over time, thus yielding a dynamic and everchanging course of disease (e.g., Galatzer-Levy et al., 2013). This symptomatic pattern also echoes Horowitz's (1997) classic theory of stress response syndromes, which conceptualized the complex ways in which posttraumatic symptoms react to and change in response to one another.

As noted, our analysis also revealed significant gender differences. Overall, women were more vulnerable to all three PTSD clusters (in two out of three waves) and MDD (in all waves). These differences are in line with a vast body of literature showing female gender to be a risk factor for both MDD and PTSD. However, it should be noted that not all previous studies have shown such a consistent pattern of gender differences across PTSD clusters. For example, in a study by Charak et al. (2014), gender differences were observed in only two of five PTSD factors (re-experiencing and "anxious arousal"). Several explanations have been suggested for women's increased vulnerability to PTSD, including stronger fear conditioning (Inslicht et al., 2013), a heavier reliance on maladaptive coping strategies (Vingerhoets and Van Heck, 1990) and more negative cognitive post-traumatic schemas and beliefs (Tolin and Foa, 2006). Higher female MDD rates were attributed to a more ruminative response style, higher exposure to negative events in the family (Hankin and Abramson, 1999) and higher neuroticism (Piccinelli and Wilkinson, 2000). Previous studies have shown that women have a higher tendency than men to monitor their environments in times of stress (e.g., Savage, 1993), a tendency which, in turn, increases their sense of threat and danger. In chronically stressful environments, this coping style may be particularly maladaptive, as threat may arise from multiple sources. This notion of increased monitoring is also in line with our finding showing a relative dominance of hyperarousal symptoms at each wave. Also, gender differences in both MDD and PTSD may be attributed to biological factors, such as a post-pubertal effect of gonadal hormones on limbic system hyperactivity, which predisposes women to potentially higher rates of both anxiety and depressive disorders (Parker and Brotchie, 2004). Finally, these reported gender differences in psychopathology may be attributed, at least in part, to different exposure patterns, and particularly to higher rates of sexual assault among women. While some studies have supported this notion (Hourani et al., 2015), others have shown that gender differences remain even when assessing men and women who reported similar types and severity of events (Tolin and Breslau, 2007; Wilhelm et al., 1998).

Women also reported more moderate recovery from both disorders with time, while men showed more consistent declines than women on 
each measure. Thus, not only are women more vulnerable to PTSD and MDD post-trauma, they also remain more symptomatic across time. As far as MDD is concerned, this finding is in line with previous studies showing a female preponderance in recurrent and chronic depression (Bracke, 1998). A unique pattern appeared for the PTSD avoidance cluster. On one hand, gender differences in avoidance were found only at W1, with women showing higher levels than men. On the other hand, avoidance was the only symptom cluster in which a quadratic trend was not observed for men, indicating a stronger recovery trend for men over time, even more so than in other PTSD clusters. These results exemplify the possible gains in conducting longitudinal analyses in psychopathology. Looking at avoidance cross-sectionally, one would not have noticed a gender difference in the temporal course of that same symptom cluster.

Finally, gender differences were observed in the longitudinal associations between PTSD symptom clusters and MDD. Whereas for women reciprocal, bidirectional associations were found between MDD and all PTSD clusters between all three waves, for men this was only partially true. In fact, the only symptom cluster in which both genders showed longitudinal bidirectional associations between PTSD and MDD was re-experiencing. Thus, for men, this was the only cluster that consistently predicted and was predicted by MDD over time. A previous study by Forbes et al. (2010) showed the re-experiencing cluster to be specifically associated with fear/phobic disorders rather than with major depression. In our sample, however, there may have been a very thin line between fear and depression, as living under chronic environmental stress may entail a sense of constant threat and danger, which in turn has the potential of hindering one's mood. Moreover, under these conditions of constant exposure, one must continuously face reminders of past traumas (e.g., gunshots, sirens, seeing and hearing about house foreclosures), which may serve an important role in preserving re-experiencing.

While paths between early MDD and later PTSD clusters were generally weaker for men than for women, an opposite gender trend was observed for the paths between early PTSD clusters and later MDD. Thus, there may be different "entry points" into post-traumatic distress for men and women. To date, very few attempts have been made to identify differential paths leading to PTSD and MDD among men and women separately. Kendler and Gardner (2014), for example, examined 1057 opposite-sex dizygotic twin pairs from a populationbased register. Over time, different factors accounted for MDD among men and women. Specifically, externalizing psychopathology and a distinct set of recent life stressors were predictive of MDD among men, but less so among women. These results seem to echo our findings that the paths connecting early PTSD to subsequent MDD were the ones most dominant among men.

\subsection{Limitations}

The present study has several limitations. First, our analyses are based on DSM-IV criteria, as this was the existing edition at the time of this study. We encourage future studies to apply similar longitudinal methodologies to assess comorbidity based on the more recent DSM-5 (Elhai et al., 2015). Second, while our longitudinal design improves the possibility of establishing causality, the present study is correlative, and therefore all inferences regarding temporal precedence of one disorder over another must be made with caution.

Despite these limitations, the present study has notable strengths. It is one of the only few studies to longitudinally assess PTSD-MDD comorbidity over time at the level of specific PTSD symptom clusters. To the best of our knowledge, it is the first study to do so among a sample of urban neighborhood residents. PTSD is often a systemic disorder, and only rarely appears in its pure form, with no comorbid conditions. Thus, it is crucial to examine its associations with other conditions with the appropriate level of complexity, by looking deeper into specific relationships between symptoms, rather than between binary diagnoses. In this respect, our findings correspond with an increasingly-growing body of literature examining post-traumatic reactions through more accurate, micro-level analyses. In a scientific era where network analysis (McNally et al., 2015) and machine learning techniques (Karstoft et al., 2015) present new options for understanding complex post-traumatic symptom profiles, the study of comorbidity may greatly benefit from following a similar route. As for clinical implications, early identification of either PTSD or MDD following trauma may be crucial in order to prevent the development of the other disorder over time. PTSD hyper-arousal may require special therapeutic attention, particularly among those living in a chronically-stressful environment. To date, several interventions have been identified as useful in treating PTSD hyperarousal. Among those are biofeedback (Reyes, 2014) and relaxation training (Carletto et al., 2016), which may be used as independent, sole interventions, or as add-ons/complementary approaches to other forms of therapy (e.g., Polak et al., 2015). Interestingly, these interventions were also demonstrated to be effective in treating major depression (Siepmann et al., 2008; Vázquez et al., 2012), perhaps indicating that arousal may be a shared target in treating both disorders. Finally, professionals are encouraged to develop more gender-specific interventions post-trauma. According to our findings, the need for such specialized interventions stems from several reasons. First and foremost, women seem to be more vulnerable to both PTSD and MDD, and thus may require more intensive treatment, as well as a more careful consideration of comorbidity. Second, men and women's distinct patterns of traumatic exposure (e.g., sexual assault vs. violent assault) often require the implementation of different treatment modules, and/or clinician's sensitivity to specific thematic elements within the trauma narrative. Third, we have hypothesized earlier about the possible emotional, cognitive and behavioral factors contributing to women's heightened post-traumatic vulnerability. Unique gender-specific factors related to coping strategies, environmental monitoring and negative cognitive schema may serve as excellent targets for psychotherapy. In recent years, specialized women's mental health clinics and programs have being opened around the world, to treat disorders as diverse as substance abuse, eating disorders and PTSD, including within the VA system (Escalona et al., 2004). Hopefully, further progress in this direction will be made in the near future.

\section{References}

American Psychiatric Association, 2000. Diagnostic and Statistical Manual: Text revised fourth ed.. American Psychiatric Association, Washington, DC.

Biehn, T.L., Contractor, A., Elhai, J.D., Tamburrino, M., Fine, T.H., Prescott, M.R. Shirley, E., Chan, P.K., Slembarski, R., Liberzon, I., Calabrese, J.R., 2013. Relations between the underlying dimensions of PTSD and major depression using an epidemiological survey of deployed Ohio national guard soldiers. J. Affect. Disord. 144 (1), 106-111.

Blake, D.D., Weathers, F.W., Nagy, L.M., Kaloupek, D.G., Gusman, F.D., Charney, D.S., Keane, T.M., 1995. The development of a clinician-administered PTSD scale. J. Trauma. Stress 8 (1), 75-90.

Bockting, C.L., Hollon, S.D., Jarrett, R.B., Kuyken, W., Dobson, K., 2015. A lifetime approach to major depressive disorder: the contributions of psychological interventions in preventing relapse and recurrence. Clin. Psychol. Rev. 41, 16-26.

Bracke, P., 1998. Sex differences in the course of depression: evidence from a longitudinal study of a representative sample of the Belgian population. Soc. Psychiatry Psychiatr. Epidemiol. 33 (9), 420-429.

Breslau, N., Kessler, R.C., Chilcoat, H.D., Schultz, L.R., Davis, G.C., Andreski, P., 1998. Trauma and posttraumatic stress disorder in the community: the 1996 detroit area survey of trauma. Arch. Gen. Psychiatry 55 (7), 626-632.

Breslau, N., Davis, G.C., Peterson, E.L., Schultz, L., 1997. Psychiatric sequelae of posttraumatic stress disorder in women. Arch. Gen. Psychiatry 54 (1), 81-87.

Breslau, N., Davis, G.C., Peterson, E.L., Schultz, L., 2000. A second look at comorbidity in victims of trauma: the posttraumatic stress disorder - major depression connection. Biol. Psychiatry 48, 902-909.

Carletto, S., Borghi, M., Bertino, G., Oliva, F., Cavallo, M., Hofmann, A., Ostacoli, L., 2016. Treating post-traumatic stress disorder in patients with multiple sclerosis: a randomized controlled trial comparing the efficacy of eye movement desensitization and reprocessing and relaxation therapy. Front. Psychol., 7.

Charak, R., Armour, C., Elklit, A., Angmo, D., Elhai, J.D., Koot, H.M., 2014. Factor structure of PTSD, and relation with gender in trauma survivors from India. Eur. J. Psychotraumatol. 5, 25547. 
Dekel, S., Solomon, Z., Horesh, D., Ein-Dor, T., 2014. Posttraumatic stress disorder and depressive symptoms: joined or independent sequelae of trauma? J. Psychiatr. Res. $54,64-69$.

Elhai, J.D., Contractor, A.A., Tamburrino, M., Fine, T.H., Cohen, G., Shirley, E., Chan P.K., Liberzon, I., Calabrese, J.R., Galea, S., 2015. Structural relations between DSM5 PTSD and major depression symptoms in military soldiers. J. Affect. Disord. 175, $373-378$.

Elwood, L.S., Hahn, K.S., Olatunji, B.O., Williams, N.L., 2009. Cognitive vulnerabilities to the development of PTSD: a review of four vulnerabilities and the proposal of an integrative vulnerability model. Clin. Psychol. Rev. 29 (1), 87-100.

Escalona, R., Achilles, G., Waitzkin, H., Yager, J., 2004. PTSD and somatization in women treated at a VA primary care clinic. Psychosomatics 45 (4), 291-296.

Fisher, D., 2015. October 29. America's Most Dangerous Cities: Detroit Can't Shake No. 1 Spot. 〈http://www.forbes.com/sites/danielfisher/2015/10/29/americas-mostdangerous-cities-detroit-cant-shake-no-1-spot/\#57bba99912c8> (accessed 21.04. 16).

Forbes, D., Parslow, R., Creamer, M., O'Donnell, M., Bryant, R., McFarlane, A., Silove, D., Shalev, A., 2010. A longitudinal analysis of posttraumatic stress disorder symptoms and their relationship with fear and anxious-misery disorders: implications for DSM-V. J. Affect Disord. 127 (1), 147-152.

Galatzer-Levy, I.R., Brown, A.D., Henn-Haase, C., Metzler, T.J., Neylan, T.C., Marmar, C.R., 2013. Positive and negative emotion prospectively predict trajectories of resilience and distress among high-exposure police officers. Emotion 13 (3), 545.

Galea, S., Ahern, J., Nandi, A., Tracy, M., Beard, J., Vlahov, D., 2007. Urban neighborhood poverty and the incidence of depression in a population-based cohort study. Ann. Epidemiol. 17 (3), 171-179.

Ginzburg, K., Ein-Dor, T., Solomon, Z., 2010. Comorbidity of posttraumatic stress disorder, anxiety and depression: a 20-year longitudinal study of war veterans. J. Affect Disord. 123 (1), 249-257.

Greaves-Lord, K., Ferdinand, R.F., Sondeijker, F.E., Dietrich, A., Oldehinkel, A.J., Rosmalen, J.G., Verhulst, F.C., 2007. Testing the tripartite model in young adolescents: is hyperarousal specific for anxiety and not depression? J. Affect. Disord. 102 (1), 55-63.

Gros, D.F., Simms, L.J., Acierno, R., 2010. Specificity of posttraumatic stress disorder symptoms: an investigation of comorbidity between posttraumatic stress disorder symptoms and depression in treatment-seeking veterans. J. Nerv. Ment. Dis. 198 (12), 885-890.

Hankin, B.L., Abramson, L.Y., 1999. Development of gender differences in depression: description and possible explanations. Ann. Med. 31 (6), 372-379.

Horesh, D., Qian, M., Freedman, S., \& Shalev, A. (2016). Differential effect of exposurebased therapy and cognitive therapy on post-traumatic stress disorder symptom clusters: A randomized controlled trial. Psychology and Psychotherapy: Theory, Research and Practice. doi: http://dx.doi.org/10.1111/papt.12103. [Epub ahead of print].

Horesh, D., Lowe, S.R., Galea, S., Uddin, M., Koenen, K.C., 2015. Gender differences in the long-term associations between posttraumatic stress disorder and depression symptoms: findings from the detroit neighborhood health study. Depress Anxiety 32 (1), 38-48.

Hu, L.T., Bentler, P.M., 1998. Fit indices in covariance structure modeling: sensitivity to underparameterized model misspecification. Psychol. Methods 3 (4), 424-453.

Horowitz, M.J., 1997. Stress Response Syndromes: PTSD, Grief, and Adjustment Disorders. Jason Aronson.

Horowitz, K., Weine, S., Jekel, J., 1995. PTSD symptoms in urban adolescent girls: Compounded community trauma. J. Am. Acad. Child. Adolesc. Psychiatry 34 (10), 1353-1361.

Hourani, L., Williams, J., Bray, R., Kandel, D., 2015. Gender differences in the expression of PTSD symptoms among active duty military personnel. J. Anxiety Disord. 29, $101-108$.

Insel, T., Cuthbert, B., Garvey, M., Heinssen, R., Pine, D.S., Quinn, K., Sanislow, C., Wang, P., 2010. Research domain criteria (RDoC): toward a new classification framework for research on mental disorders. Am. J. Psychiatry 167 (7), 748-751.

Inslicht, S.S., Metzler, T.J., Garcia, N.M., Pineles, S.L., Milad, M.R., Orr, S.P., Neylan, T.C., 2013. Sex differences in fear conditioning in posttraumatic stress disorder. J. Psychiatr. Res. 47 (1), 64-71.

Itzhaky, L., Levin, Y., Fingerhut, H., Solomon, Z., 2014. Depression and PTSD comorbidity: what are we missing? J. Depress. Anxiety 3 (4), 164.

Karstoft, K.I., Galatzer-Levy, I.R., Statnikov, A., Li, Z., Shalev, A.Y., 2015. Bridging a translational gap: using machine learning to improve the prediction of PTSD. BMC Psychiatry 15 (1), 30.

Kashdan, T.B., Morina, N., Priebe, S., 2009. Post-traumatic stress disorder, social anxiety disorder, and depression in survivors of the Kosovo War: experiential avoidance as a contributor to distress and quality of life. J. Anxiety Disord. 23 (2), 185-196.

Kendall-Tackett, K.A., 2000. Physiological correlates of childhood abuse: chronic hyperarousal in PTSD, depression, and irritable bowel syndrome. Child Abus. Negl. 24 (6), 799-810

Kendler, K.S., Gardner, C.O., 2014. Sex differences in the pathways to major depression: a study of opposite-sex twin pairs. Am. J. Psychiatry 171, 426-435.

Koren, D., Arnon, I., Klein, E., 2001. Long term course of chronic posttraumatic stress disorder in traffic accident victims: a three-year prospective follow-up study. Behav. Res. Ther. 39 (12), 1449-1458.

Kroenke, K., Spitzer, R.L., 2002. The PHQ-9: a new depression diagnostic and severity measure. Psychiatr. Ann. 32 (9), 509-521.

Kroenke, K., Spitzer, R., Williams, J., 2001. The PHQ-9: validity of a brief depression severity measure. J. Gen. Intern. Med. 16 (9), 606-613.

Kulka, A.R., Schlenger, W.E., Fairbank, J.A., Hough, R.L., Jordan, B.K., Marmar, C.R., Weiss, D.S., 1990. Trauma and the Vietnam War Generation: Report of Findings from the National Vietnam Veterans Readjustment Study. Brunner/Mazel.

Lovallo, W.R., 1997. Stress and Health: Biological and Psychological Interactions. Sage publications.

McNally, R.J., Robinaugh, D.J., Wu, G.W., Wang, L., Deserno, M.K., Borsboom, D., 2015. Mental disorders as causal systems a network approach to posttraumatic stress disorder. Clin. Psychol. Sci. 3 (6), 836-849.

Myers, H.F., Wyatt, G.E., Ullman, J.B., Loeb, T.B., Chin, D., Prause, N., Zhang, M., Williams, J.K., Slavich, G.M., Liu, H., 2015. Cumulative burden of lifetime adversities: trauma and mental health in low-SES African Americans and Latino/as. Psychol. Trauma 7 (3), 243.

Norris, F.H., Murphy, A.D., Baker, C.K., Perilla, J.L., 2004. Postdisaster PTSD over four waves of a panel study of Mexico's 1999 flood. J. Trauma. Stress 17 (4), 283-292.

O’Donnell, M.L., Creamer, M., Pattison, P., 2004. Posttraumatic stress disorder and depression following trauma: understanding comorbidity. Am. J. Psychiatry 161, 1390-1396.

Parker, G.B., Brotchie, H.L., 2004. From diathesis to dimorphism: the biology of gender differences in depression. J. Nerv. Ment. Dis. 192 (3), 210-216.

Piccinelli, M., Wilkinson, G., 2000. Gender differences in depression Critical review. Br. J. Psychiatry 177 (6), 486-492.

Polak, A.R., Witteveen, A.B., Denys, D., Olff, M., 2015. Breathing biofeedback as an adjunct to exposure in cognitive behavioral therapy hastens the reduction of PTSD symptoms: a pilot study. Appl. Psychophysiol. Biofeedback 40 (1), 25-31.

Post, L.M., Zoellner, L.A., Youngstrom, E., Feeny, N.C., 2011. Understanding the relationship between co-occurring PTSD and MDD: symptom severity and affect. J. Anxiety Disord. 25 (8), 1123-1130.

Price, M., van Stolk-Cooke, K., 2015. Examination of the interrelations between the factors of PTSD, major depression, and generalized anxiety disorder in a heterogeneous trauma-exposed sample using DSM 5 criteria. J. Affect. Disord. 186, 149-155.

Reyes, F.J., 2014. Implementing heart rate variability biofeedback groups for veterans with posttraumatic stress disorder. Biofeedback 42 (4), 137-142.

Rubacka, J.M., Schmeidler, J., Nomura, Y., Luthra, R., Rajendran, K., Abramovitz, R., Chemtob, C.M., 2008. The relationship between PTSD arousal symptoms and depression among mothers exposed to the World Trade Center attacks. J. Nerv. Ment. Dis. 196 (6), 504-507.

Rubin, D.B., 1987. Multiple Imputation for Nonresponse in Surveys 81. John Wiley \& Sons.

Savage, I., 1993. Demographic influences on risk perceptions. Risk Anal. 13 (4), $413-420$.

Schindel-Allon, I., Aderka, I.M., Shahar, G., Stein, M., Gilboa-Schechtman, E., 2010. Longitudinal associations between post-traumatic distress and depressive symptoms following a traumatic event: a test of three models. Psychol. Med. 40 (10), 1669-1678.

Seligowski, A.V., Rogers, A.P., Orcutt, H.K., 2016. Relations among emotion regulation and DSM-5 symptom clusters of PTSD. Personal. Individ. Differ. 92, 104-108.

Siepmann, M., Aykac, V., Unterdörfer, J., Petrowski, K., Mueck-Weymann, M., 2008. A pilot study on the effects of heart rate variability biofeedback in patients with depression and in healthy subjects. Appl. Psychophysiol. Biofeedback 33 (4), 195-201.

Solomon, Z., Horesh, D., Ein-Dor, T., 2009. The longitudinal course of posttraumatic stress disorder symptom clusters among war veterans. J. Clin. Psychiatry 70 (6), 837-843.

Tolin, D.F., Breslau, N., 2007. Sex differences in risk of PTSD. PTSD Res. Quarterly 18 (2), $1-8$.

Tolin, D.F., Foa, E.B., 2006. Sex differences in trauma and posttraumatic stress disorder: a quantitative review of 25 years of research. Psychol. Bull. 132 (6), 959.

Uddin, M., Aiello, A.E., Wildman, D.E., Koenen, K.C., Pawelec, G., de Los Santos, R., Goldmann, E., Galea, S., 2010. Epigenetic and immune function profiles associated with posttraumatic stress disorder. Proc. Natl. Acad. Sci. USA 107 (20), 9470-9475.

Vázquez, F.L., Torres, A., Blanco, V., Díaz, O., Otero, P., Hermida, E., 2012. Comparison of relaxation training with a cognitive-behavioural intervention for indicated prevention of depression in university students: a randomized controlled trial. J. Psychiatr. Res. 46 (11), 1456-1463.

Vingerhoets, A.J., Van Heck, G.L., 1990. Gender, coping and psychosomatic symptoms. Psychol. Med. 20 (01), 125-135.

Weathers, F.W., Ford, J., 1996. Psychometric review of PTSD checklist (PCL-C, PCL-S, PCL-M, PCL-PR). Meas. Stress Trauma, Adapt., 250-251.

Wilhelm, K., Parker, G., Dewhurst, J., 1998. Examining sex differences in the impact of anticipated and actual life events. J. Affect. Disord. 48 (1), 37-45. 